\title{
Erratum to: Architecture and Design of Distributed Embedded Systems
}

\author{
Bernd Kleinjohann \\ University of Paderborn / C-LAB, Germany
}

\section{Erratum to: \\ B. Kleinjohann (Ed.) \\ Architecture and Design of Distributed \\ Embedded Systems \\ DOI: $10.1007 / 978-0-387-35409-5$}

The book was inadvertently published with an incorrect name of the copyright holder. The name of the copyright holder for this book is: (c) IFIP International Federation for Information Processing. The book has been updated with the changes. 